\title{
A Competition-based Algorithm for Routing Discovery and Repair in Large-scale VANET
}

\author{
Cheng $\mathrm{Wu}^{1}$, Lujie Wang ${ }^{1}$ and Yiming Wang ${ }^{1}$ \\ ${ }^{1}$ School of Urban Rail Transportation, Soochow University \\ 8 Jixue Road, Suzhou, Jiangsu 215011 - PRC \\ [e-mail: cwu@suda.edu.cn] \\ ${ }^{2}$ School of Urban Rail Transportation, Soochow University \\ 8 Jixue Road, Suzhou, Jiangsu 215011 - PRC \\ [[e-mail: ymwang@suda.edu.cn] \\ *Corresponding author: Yiming Wang
}

Received June 3, 2017; revised July 26, 2017; accepted August 9, 2017; published December 31, 2017

\begin{abstract}
Vehicular Ad Hoc Networks (VANET) in the large-scale road section usually have typical characteristics of large number of vehicles and unevenly distribution over geographic spaces. These two inherent characteristics lead to the unsatisfactory performance of VANETs. This poor performance is mainly due to fragile communication link and low dissemination efficiency. We propose a novel routing mechanism to address the issue in the paper, which includes a competition-based routing discovery with priority metrics and a local routing repair strategy. In the routing discovery stage, the algorithm uses adaptive scheme to select a stable route by the priorities of routing metrics, which are the length of each hop, as well as the residual lifetime of each link. Comparisons of different ratios over link length and link stability further show outstanding improvements. In the routing repair process, upstream and downstream nodes also compete for the right to establish repair process and to remain as a member of the active route after repair. Our simulation results confirm the improved performance of the proposed algorithm.
\end{abstract}

Keywords: Vehicular Ad Hoc Network, Large-scale road section, Routing discovery and repair, Routing metric 


\section{Introduction}

As a special kind of ad-hoc network designed for transport sector, VANET is a promising road-specific application-oriented solution for various real-time scenarios. Its essence is a highly mobile Ad-hoc network (MANET) in which each moving vehicle works as a wireless node to communicate with its neighbor vehicles or road-side devices without the client and server structure. It is proven to be efficient to deal with the challenge of low communication quality by high-speed mobility, intermittent connectivity and diverse topology[1] [2] [3].

In the past few decades, so many routing protocols in VANET have been proposed for the establishment of stable and robust routing. Most of them are classified as topology-based routing, including reactive routing, proactive routing and hybrid routing, and position-based routing.In reactive routing, the route to the destination node is established only when required. It broadcasts RREQ packets till the destination sends a RREP packet back to the source node. Typical studies are Dynamic Source Routing (DSR) [4], Ad hoc on-demand distance vector (AODV) [5] and its variation[2] [6] etc. In proactive routing, the nodes in the network are maintaining a routing table. Each node sends regularly a "hello" packet to its neighbors for showing the presence. The nodes receiving the packet update their routing tables for tracking the changing of the network. Examples in the category are Destination-sequenced Distance Vector (DSDV) [7] and Optimized Link State Routing (OLSR) [8].Typical hybrid routing divides the network into two areas: local and global. In order to achieve greater efficiency and scalability, Zone Routing Protocol combines the local active routing protocol with the global reactive routing protocol to minimize routing overhead and delay due to the route discovery process [9]. The current routing path used by the network still needs to be maintained by combining two policies, since it limits the topology change within a given amount of time [3].In position-based routing, each node in the network knows its own position using positioning device, such as GPS, and It floods RREQ packets in a restrict region by priori geographic information. Such examples include Distance Routing Effect Algorithm for Mobility (DREAM) [10]and Location-aided routing (LAR) [11].

As to the route repair process, the traditional AODV provides its typical route repair solution. If the break is not far away from destination node, the upstream node launches local repair for the destination as opposed to source repair [12]. In some circumstances, this achieves the purpose of saving the time for route rediscovery and decreasing routing overhead. Azzuhri et al. define Local Repair Threshold parameter to determine the distance that the breaks occur from the source node along the whole route to help make a choice between source repair and local repair[8] [13]. However, in the local repair process above, the destination is always the same as that of the original route. The reestablishment of the rest route that linked original leads to a waste of network resource and an increase in 
transmission delay. Furthermore, the literatures [14] [15] above have a single-way route repair from upstream node to downstream node in common. In opposite, the local repair process suggested by Zheng et al. is from downstream node to upstream node. This leaves out the process of broadcasting RREQ from upstream node and contributes to the reduction of route repair delay and network overhead [16] [13]. This is still a single-way repair but in the reverse direction. As the route break can be caused by the movement of upstream node as well as downstream node, using simplex local repair without eliminating intensely-moving node would lead to a fragile link after repair.

The routing discovery and repair process mentioned above provides a rich solution for VANET, and also shows certain performance within a limited network scale. However, recent development shows that more and more VANET studies are including more vehicles and covering wider region [17] [18] [19]. In fact, from the point of view of transportation field, most of traffic flows in urban road networks are naturally large-scale systems with gigantic topologies and enormous devices [20] [21]. Studying such a large-scale VANET in urban environment is necessary due to two of unique performance-related characteristics:

- The real-world urban road network spans over wide geographic regions: the expansion of network scale unavoidably increases the complexity of vehicle network topology and the disordering of vehicle movements, making data links much more frequently fragile [17].

- The network has a large amount of vehicles and road-side devices: more connection nodes in the network leads to the increase of route overhead and setup time. The cost of routing discovery and repair also distinctly goes up [21].

- The type of data service between the vehicles is also undergoing significant changes. It gradually evolves from simple low-byte texts to large-capacity video [22]. Video applications with high user satisfaction are becoming an important part of V2V communications. This change puts forward higher requirements for the routing topology of vehicle communication [23].

In order to reduce the negative impact of the expansion of topology and the increase of moving vehicles, choosing optimal next-hop nodes in a competitive manner for route establishment is clearly a good way [24]. The competition process should be based on new routing metric system. Therefore, we focus our research on a routing algorithm for solving large-scale VANET, called Competition-based Routing Algorithm with new integrated routing metric. To improve link stability, both link length and residual lifetime are considered into routing metric. If the link breaks, the more stable node between upstream and downstream ones would be selected to initialize route repair. The other one is discarded due to high occurrence of another link breakage. It helps alleviate adverse impact of repeated link breaks on the network performance. 
The rest of paper is organized as below. Section 2 describes how to design the integrated routing metric to compete next hop in the route establishment process and presents the simulation results. In section 3, the mechanism of competition is applied in the route repair process between upstream and downstream nodes in the route maintenance process. Finally, some concluding remarks are given in Section 4.

\section{Routing Discovery Process}

In VANET, forwarding area refers to a specific region within its communication range designated by previous node. All nodes within this region enable to forward RREQs, while the others outside have to omit them directly. In Fig. 1, the shaded area illustrates the forwarding area of previous node $N_{s}$. Intermediate nodes $N_{i_{1}}, N_{i_{2}}$ and $N_{i_{3}}$ are the neighbor nodes of $N_{s}$ in its communication range. $N_{d}$ is the destination of data packets. $R$ is the radius of $N_{s}$ 's communication range. $N_{i_{1}}$ and $N_{i_{2}}$ are in the forwarding area, which are qualified for forwarding RREQs towards $N_{d} . N_{i_{3}}$ is out of the forwarding area, which drops RREQs.

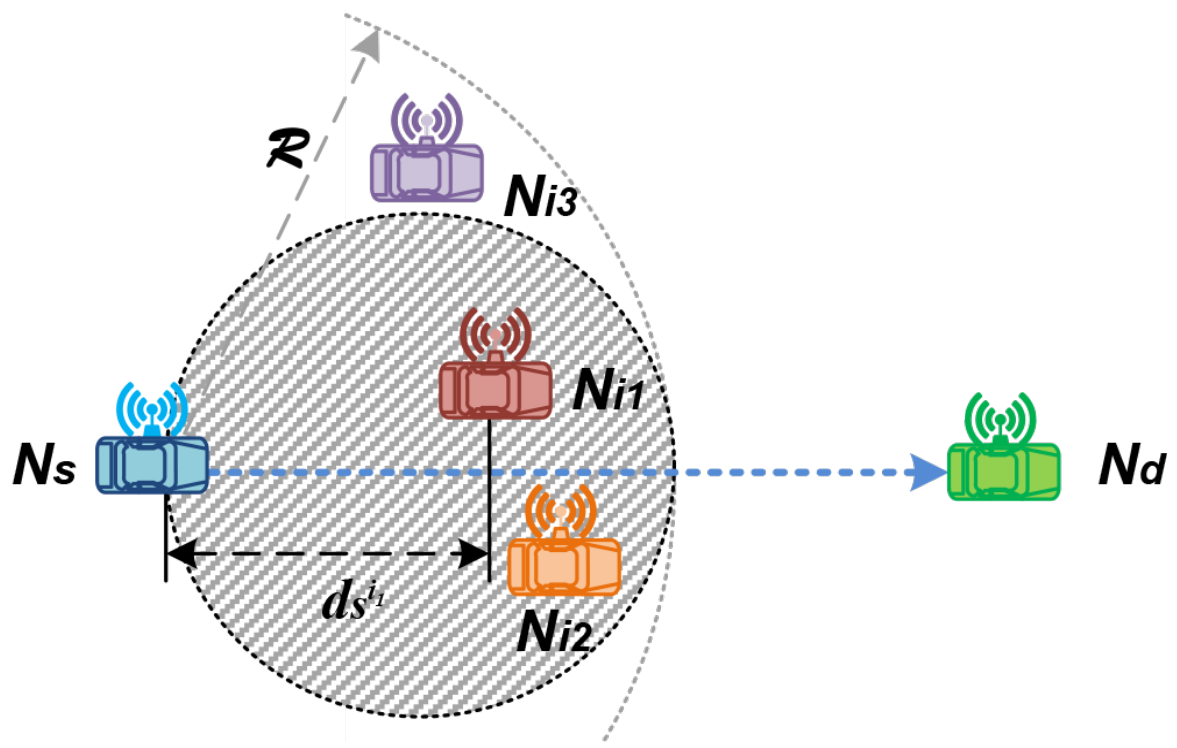

Fig. 1. Forwarding area

The forwarding area in the proposed protocol can be seen as a circular region with the radius of $R / 2$. Once a node forwards packets, the others in the forwarding area can overhear and respond. All nodes in the forwarding area must compete to broadcast RREQs based on a competing parameter $\omega$. Each candidate calculates its own forwarding delay $t_{d}$ by Equation 1 .

$$
t_{d}=\text { max_delay } \times(1-\omega)
$$

max_delay implies the transmission time of node. It is an estimated value of a packet's one-hop traversal time, including queuing delay, interrupt processing time and propagation 
time. $\omega$ is used to quantify the competitiveness of each node using the routing metric. If the value of $\omega$ over one node is maximal among all candidates, the path from $N_{s}$ to the node is believed to be the most stable and suitable link. The node with the smallest forwarding delay can naturally forward the RREQ first. Once the other nodes in the same forwarding area have overheard the RREQs packet from the first forwarded node, they omit the forwarding request of source node $N_{s}$ immediately.

\subsection{Integrated Routing Metric for Competition}

In our proposal, the routing metric can be used to determine the competing parameter $\omega$. How to design the routing metric is the key for routing establishment. In large-scale VANET, whether a route is optimal is depended on both link length and link residual lifetime. Hence, we consider the interleaving effect of link residual lifetime and the least number of hops.

We assume that all vehicular nodes have their own geographical information such as location, direction and speed, which may be acquired by GPS. The movement of any node is constant at the current instant. The link residual lifetime can be illustrated in Fig. 2. Here $N_{s}$ is the previous node, $N_{d}$ is the destination node and $N_{i}$ is one of the candidates for routing in the forwarding area, which gets RREQs from $N_{s}$.

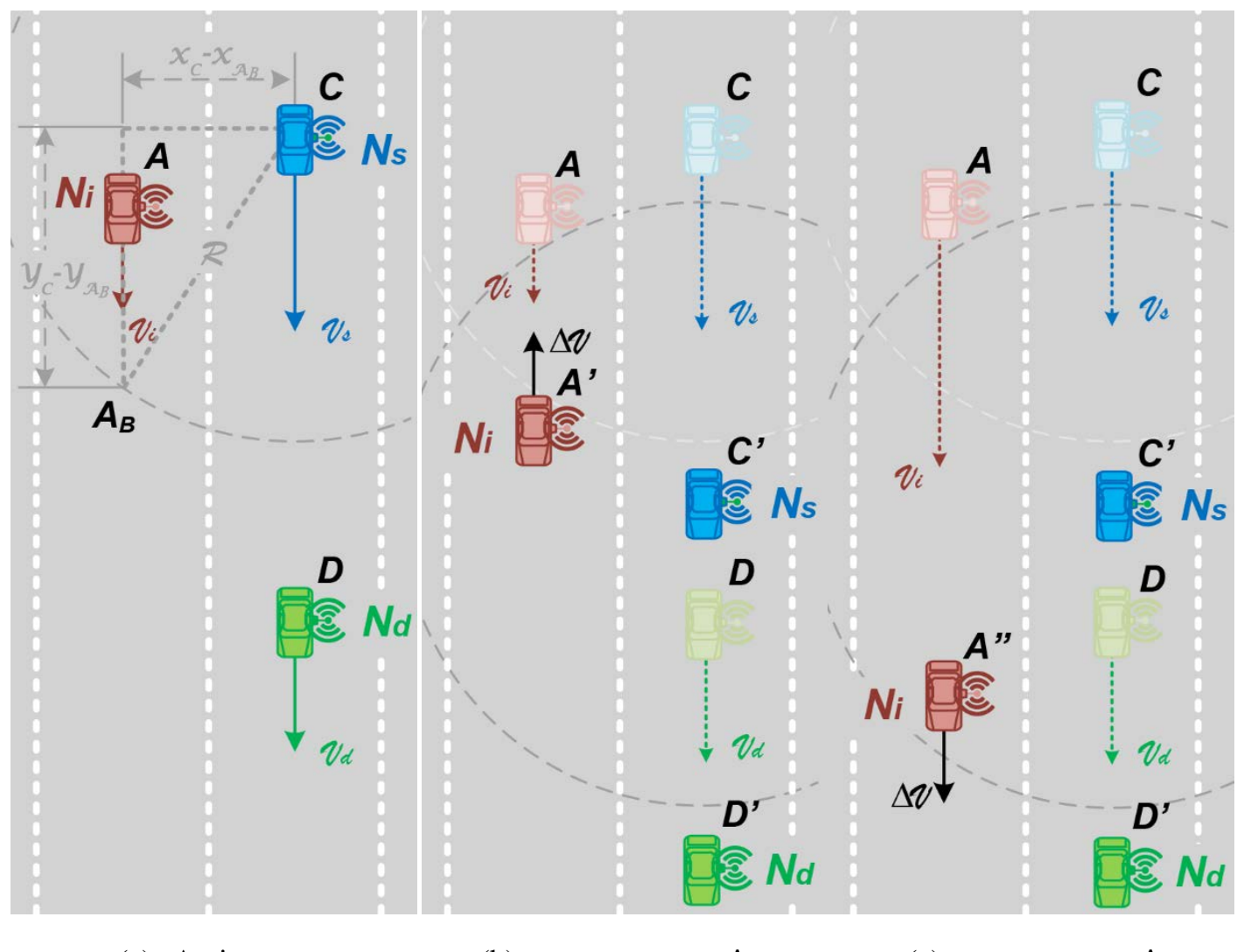

(a) At time $t$

(b) $v_{s}>v_{i}$ at $t+\Delta t$

(c) $v_{s}<v_{i}$ at $t+\Delta t$

Fig. 2. The calculation of link residual lifetime 
Fig. 2(a) shows that at the initial moment $t, N_{S}$ is at Point $C\left(x_{C}, y_{C}\right), N_{d}$ is at Point $D\left(x_{D}, y_{D}\right)$ and $N_{i}$ is at Point $A\left(x_{A}, y_{A}\right)$, which is located ahead of $N_{S}$ on the right. After an instant $\Delta t$, if $N_{i}$ keeps a constant speed, then the relative movement of $N_{i}$ to $N_{S}$ has two cases. Each case is characterized by different speed conditions. One case is shown in Fig. 2(b). The speed $v_{S}$ of $N_{s}$ is faster than $v_{i}$ of $N_{i}$. At the moment $t+\Delta t, N_{s}$ moves from C to C'. $N_{i}$ moves from $A$ to $A^{\prime}$, which is on the rear right of $N_{s}$. During the moving, $N_{i}$ first gets closer to $N_{s}$, and then goes far away from it.Another case is shown in Fig. 2(c). $v_{S}$ is slower than $v_{i}$. After $\Delta t, N_{s}$ moves to C'. $N_{i}$ gradually moves far away from $A$ to $A^{\prime \prime}$. If $N_{i}$ moves outside $N_{s}$ 's communication area, the established link between these two nodes would break.

Assume $A_{b}$ is exactly at the boundary of $N_{s}$ 's communication area. The distance between $A$ and $A_{b}$, denoted as $D_{r}$, is the remainder distance that $N_{i}$ can move before link breakage. $T_{r}$ is the link residual lifetime, which is the remainder time left for communicating. Hence we have

$$
T_{r}=\frac{D_{r}}{\left|v_{s}-v_{i}\right|}
$$

Table 1 shows the values of $D_{r}$ and $T_{r}$ under the cases above. Integrating $T_{r}$ in the cases, we can deduce the general formula of link residual lifetime:

$$
T_{r}=\frac{\sqrt{\left(v_{s}-v_{i}\right)^{2}\left[R^{2}-\left(x_{C}-x_{A_{b}}\right)^{2}\right]}+\left(v_{s}-v_{i}\right)\left(y_{A}-y_{C}\right)}{\left(v_{s}-v_{i}\right)^{2}}
$$

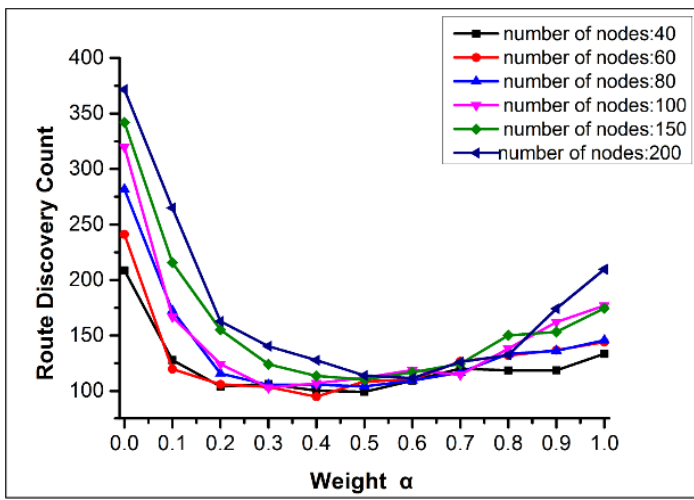

(a)Route Discovery Count.

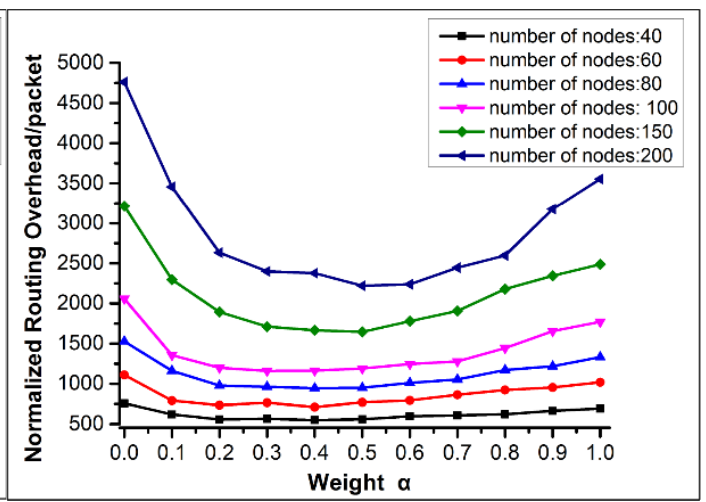

(b)Normalized Routing Overhead

Fig. 3. Effect of weight $\alpha$

Table 1. The calculation of $D_{r}$ and $T_{r}$

\begin{tabular}{|c|c|c|}
\hline \multicolumn{2}{|c|}{$\boldsymbol{D}_{\boldsymbol{r}}$} & $\boldsymbol{T}_{\boldsymbol{r}}$ \\
\hline \hline$v_{s}>v_{i}$ & $\sqrt{R^{2}-\left(x_{C}-x_{A_{b}}\right)^{2}}+\left(y_{A}-y_{C}\right)$ & $\frac{D_{r}}{v_{s}-v_{i}}$ \\
\hline$v_{s}<v_{i}$ & $\sqrt{R^{2}-\left(x_{C}-x_{A_{b}}\right)^{2}}-\left(y_{A}-y_{C}\right)$ & $\frac{D_{r}}{v_{i}-v_{s}}$ \\
\hline
\end{tabular}


Our proposed routing metric also takes route length into account, except link residual lifetime. As shown in Fig. 1, $d_{s}^{i_{1}}$ is the projection distance of $N_{s}$ to $N_{i_{1}}$. It represents the move towards $N_{s}$ away from $N_{i_{1}}$. The node with larger $d_{s}^{i_{1}}$ is relatively closer to the destination. Obviously, the route over the nodes with the largest $d_{s}^{i}$ has the fewest hops.

Routing metric is defined to integrate $d_{s}^{i}$ and $T_{r}$ together by competing parameter $\omega$, and the weight $\alpha$ is used to adjust the bias on these two parameters, as shown in Equation 4.

$$
\omega=(1-\alpha) \frac{T_{r}}{T_{\max }}+\alpha \frac{d_{s}^{i}}{R}
$$

where $T_{\max }$ is the maximal value of residual lifetime, and $T_{r} \leq T_{\max }$. If $\alpha$ is appropriate, an optimal route with relatively fewer hops and greater stability can be selected. At the time, the competing parameter $\omega$ must be the optimal value.

\subsection{Scenario Model and Simulation Analysis}

Scenario Model Our experiment simulation is from one of typical urban traffic scenarios. When the traffic light turns green, all waiting vehicles start to move forward, and may stop at next intersection if the red light is on. Between the two intersections, all vehicles in different lanes move to the same direction with different speeds and constitute a temporary mobile ad-hoc network. Each vehicle can be seen as the source or the forwarder of data packets. Nodes switch from go-straight state to change-lane state through cycles until they reach the second intersection. When a simulation starts, the sources of five pairs of vehicle nodes generate data packets within 30 seconds. All nodes at the moment move forward and cross the first intersection. They are scattering on the lanes. Once the pair reach the second intersection, the communication between them does stop. When all of 5 pairs reach the second intersection, the simulation ends. Simulation parameters are in Table 2. $T_{\max }$ is set 80s. According to the simulation, the probability that a link survives till $80 \mathrm{~s}$ is close to 0 . Note that the residual

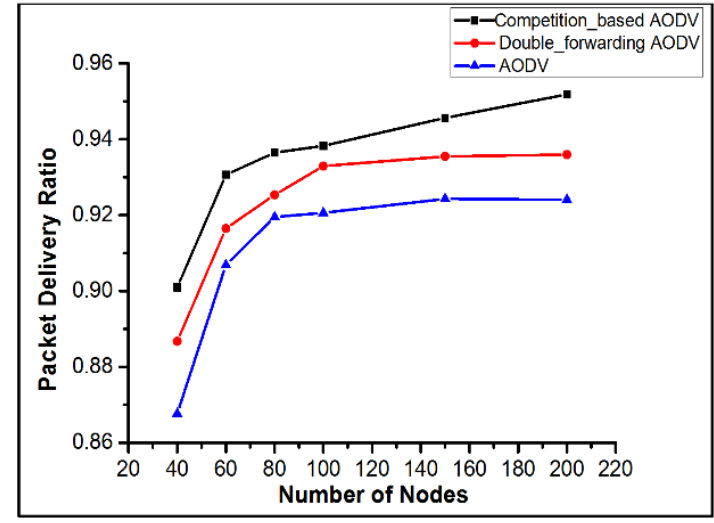

(a)Packet Delivery Radio.

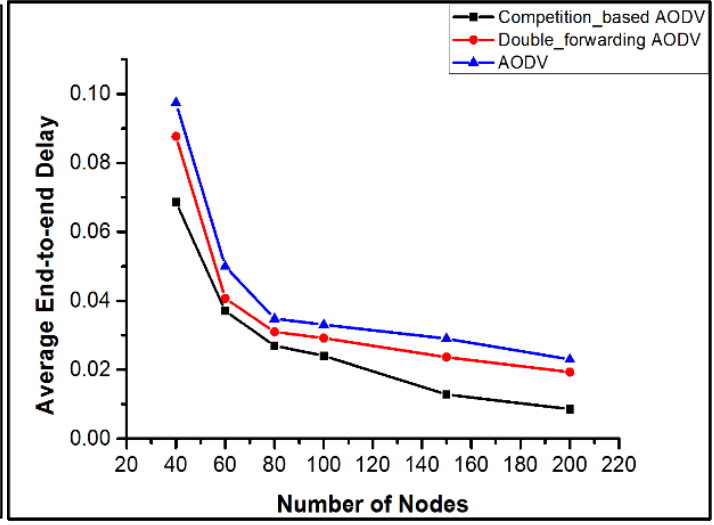

(b)Average End-to-End Delay.

Fig. 4. Performance of Competition-based at $\alpha=0.5$, D-AODV and AODV 
Table 2. Simulation Patameters

\begin{tabular}{|c|c|}
\hline Patameters & Valne \\
\hline \hline Simulation area & $37.3 \mathrm{~m} \times 2000 \mathrm{~m}$ \\
\hline Numbei of lanes & 10 \\
\hline Traffic type & $40,60,80,100,150,200$ \\
\hline Packet rate & Constant Bit Rate(CBR) \\
\hline Data Packet Size & $5 \mathrm{packets} / \mathrm{sec}$ \\
\hline Node speed & $10 \mathrm{~km} / \mathrm{h} \sim 80 \mathrm{~km} / \mathrm{h}$ \\
\hline Communication range (R) & $250 \mathrm{~m}$ \\
\hline$T_{\text {max }}$ & $80 \mathrm{sec}$ \\
\hline max_delay & $0.04 \mathrm{sec}$ \\
\hline MAC & $802.11 \mathrm{~b}$ \\
\hline
\end{tabular}

lifetime is meaningless if its value is larger than 80 s.

Effect of Weight a The optimized routing metric includes two parameters, link length $d_{s}^{i}$ and link residual lifetime $T_{r}$. We investigate their combinations, and deduce the competing parameter $\omega$ and its corresponding forwarding delay $t_{d}$. Among all candidate nodes, the one with the maximal competing parameter can become a forwarding node with the highest priority. Various $\alpha$ means different bias to two competition parameters. Fig. 3 shows the effect of weight $\alpha$ changing from 0 to 1 to network performance. Two criterions, route discovery count in Fig. 3(a) and routing overhead in Fig. 3(b), are evaluated. The results indicate that both of them decrease at the beginning, and then increase slowly. Their minimal values exist at $\alpha=0.5$. It implies that residual lifetime and link length effectively work together for achieving a relatively stable route with less rediscovery and lower overhead. We note that if $\alpha=0$, the node with the maximal link residual lifetime would forward RREQ in every hop. Any change on the speed or direction of node may change $\omega$ greatly, causing unstable. If $\alpha=1$, the further node from $N_{s}$ has higher priority to forward packets. Small relative movement in any pair may cause link break. The effect of $\alpha$ proves that integrating $d_{s}^{i}$ and $T_{r}$ in route metric greatly contributes to network performance.

Analysis and Comparison We further investigate three performance criterions such as packet delivery rate, average end-to-end delay and normalized routing overhead in our proposed competition-based scheme. It is compared with Double-forwarding AODV (D-AODV) [25] and AODV. Here $\alpha$ is set 0.5 for better performance. Consider the reliability of simulation, all results are statistically obtained by averaging 50 runs. All values in 50 runs with different seeds.

Packet Delivery Ratio: In Fig. 4(a), the packet delivery ratios of AODV, double-forwarding AODV and competition-based AODV protocols gradually increase as the node group 
expands. It is because the growth of the number of nodes brings in better network connectivity. Further, it indicates that the packet delivery ratio of competition-based AODV is clearly larger than that of double-forwarding AODV in the case of various number of nodes. The result implies that the competition-based AODV is superior when the number of nodes increases. This can be explained by the fact that the prediction of route lifetime in competition-based AODV does eliminate frequent link breaks and improve packet delivery ratio. In fact, the predictive mechanism of route lifetime provides a very efficient and very direct transit node filtering process. Using a routing node with a longer lifetime can reduce unnecessary routing switching overhead.

Average End-to-end Delay: It includes all possible delays such as route discovery latency, queuing time and propagation delay. Fig. 4(b) indicates the relationship between average end-to-end delay and the number of nodes. The results show that average end-to-end delay gradually decreases when vehicle density increases. It is explained by the fact that each node has more neighbors to find an optimal route. These results indicate that competition-based scheme has the ability to select further node with longer residual lifetime because it takes both link length and residual lifetime in account, not only link length as in D-AODV. Thus its delay is definitely shorter than those AODVs. It is really worthy in VANET environment with large-scale topology and enormous vehicles.

\section{Routing Repair Process}

In large-scale VANET, using Competition-based route discovery with routing metrics can reduce the negative impact of larger topology and more moving vehicles. However, the kind of "larger" and "more" still cause the fickleness and volatility of the whole VANET. Hence, in practice, repairing the breakage of links as soon as possible is more meaningful. One feasible approach is to limit route repair to occur around the surrounding area of breakage, which may shorten route discovery process. We therefore define to launch local repair at only upstream node, as $N_{\text {up }}$, or downstream node, as $N_{\text {down }}$. In the strategy, $N_{u p}$ and $N_{\text {down }}$ compete the right of route repair according to their own stability indexes. The more stable node initiates a route repair and another is not allowed to involve in this route repair.

\subsection{Competition between Upstream and Downstream Nodes}

In the route discovery, the link residual lifetime is used as one parameter for link stability. However, it is obvious that the data acquired for computing residual life is outdated and inaccurate. Therefore, using the stability index $\beta$ to evaluate the stability of upstream and downstream nodes is a feasible solution.A stability index, denoted as $\beta$, is to evaluate the stability of $N_{u p}$ or $N_{\text {down }}$.

Now we define a set of nodes: $S_{N_{j}}^{t}=\left\{N_{i} \mid N_{i}\right.$ is a neighbor of Node $N_{j}$ at time $\left.t, i=1,2, \ldots\right\}$. 


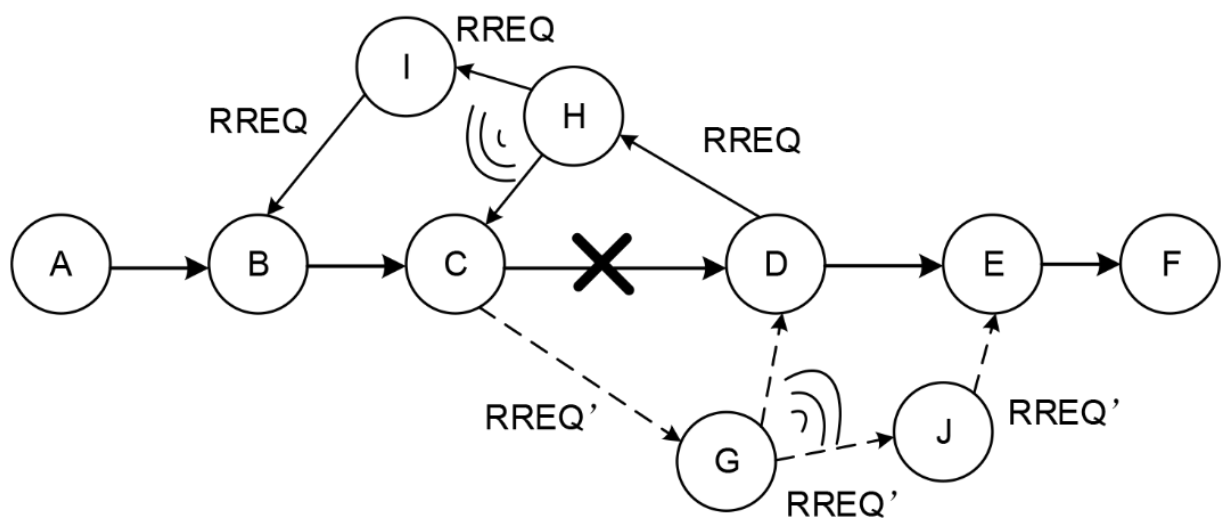

(a) The break locates at the Middle of the Route

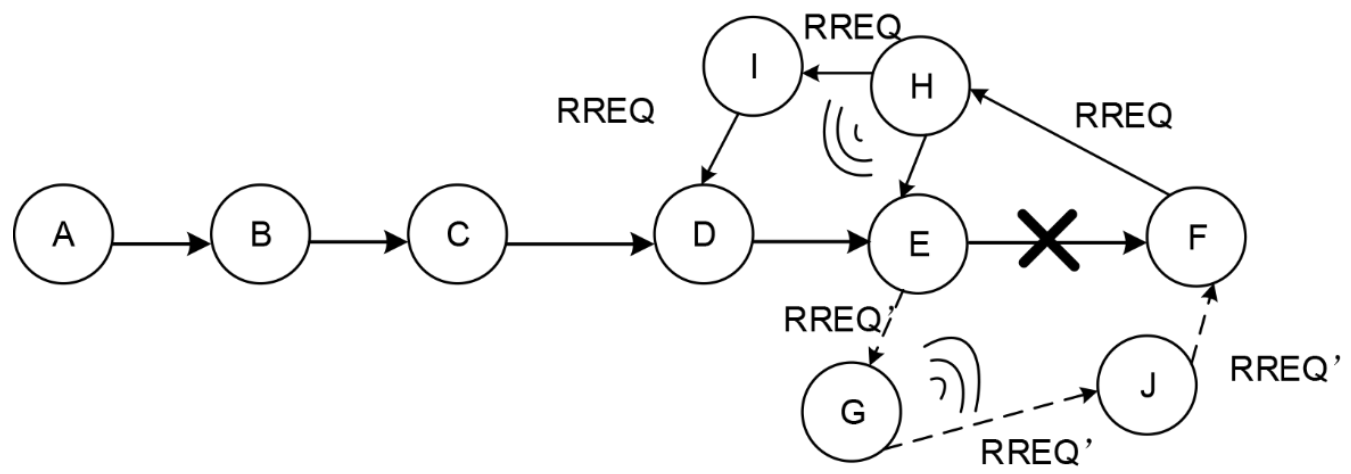

(b) the break locates at the End of the Route

Fig. 5. Route repair in different conditions

At continuous instants $t_{1}$ and $t_{2}$, we have $S_{N_{j}}^{t_{1}}$ and $S_{N_{j}}^{t_{2}}$ at $N_{j}$. The stability index of $N_{j}$ is the loss rate of neighbors of $N_{j}$ from $t_{1}$ to $t_{2}$ compared to the total number of neighbors at moment $t_{1}$.

$$
\beta_{N_{j}}=\left(\left|S_{N_{j}}^{t_{1}}\right|-\left|\left(S_{N_{j}}^{t_{1}} \cap S_{N_{j}}^{t_{2}}\right)\right| /\left|S_{N_{j}}^{t_{1}}\right|\right)
$$

The smaller $\beta$ is, the more stable the node is. Here the update cycle of stability index is $5 \mathrm{~s}$. It means each node calculates its stability index $\beta$ in every five seconds. Then the index is added in the "Hello" packet and broadcasted to its neighbors. So every neighbor receiving copies $\beta$ to the entry of neighboring node list so that the value of $\beta$ in the list is updated.

\subsection{Local Repair with Competition}

When a link breaks, a local repair starts in order to quickly reconnect the route and continue data packets transmission. For instance, in Fig. 5, a path of nodes $N_{A}-N_{B}-N_{C}-N_{D}-$ $N_{E}-N_{F}$ is the original route. A link break occurs between $N_{C}$ and $N_{D} . N_{C}$ and $N_{D}$ first compare their stability index that have been updated before, and then figure out which is 
more stable. If $\beta_{N_{C}}<\beta_{N_{D}}$, that is, the upstream node $N_{C}$ is more stable than the downstream node $N_{D}, N_{C}$ is entitled to launch a route repair. It is called " $N_{u p}$ Repair". Otherwise, $N_{D}$ launches a route repair, which is called " $N_{\text {down }}$ Repairs".

Different from the route discovery process, the destination node here is the next-to-next hop along the route from the fracture. In that case, the unstable one between $N_{u p}$ and $N_{\text {down }}$ would be kicked out, which can reduce the risk to be broken again and improve the efficiency of route repair. The route is repaired by sending route repair request packets in the broadcasting way from break end within three hops in maximum. Note that a traditional routing table only record the information of adjacent upstream and downstream nodes. It is not enough for our proposed algorithm. The new routing table need to add the "hop after next" entry, and the value of "hop after next" can be obtained at the time of route establishment.

Upstream Node Repair In Fig. 5(a), node $C$ is more stable than node $D$, node $C$ initiates upstream repair. Node $C$ seeks for the route entry indexed by the address of node $F$ and figures out whether its parameter "hop after next" is valid. If it is valid, node $C$ broadcasts RERREQ (route repair request packet) destined to "hop after next" node $E$ with the maximal 3 hops. Assume that RERREQ packet goes through node $G$ and $J$ and finally reaches node $E$. Node $E$ looks into the route table. If the path from node $E$ to node $F$ is valid, then node $E$ replies RERREP(route repair reply packet). Both node $G$ and $J$ update their routes to node $E$ as well as to node $F$. When RERREP reaches node $C$, the reverse path $C-G-J-E$ establishes and replaces the failed path. If the parameter "hop after next" is invalid, then upstream node $C$ switches to the traditional local repair method and send RERREQ to the original destination node $F$. When sending RERREQ, node $C$ sets a timer at the same time. If node $C$ cannot receive RERREP in a certain duration, which means local route repair fails, then node $C$ sends route error message to source node $A$ and requires for the source repair. When node $C$ receives data packets during local repair, it first stores the packet and waits to forward it on the fixed route.

Downstream Node Repair If node $D$ is more stable than node $C$, node $D$ initiates the downstream repair. The node $D$ uses a similar method in downstream node repair as node $C$ in upstream node repair. Node $D$ broadcasts RERREQ destined to node $B$. New path $B$ - $I-H-D$ establishes and replaces the failed path. Node $G$ and $J$ update their routes to node $E$ and node $F$. Note that node $C$ needs to send a one-hop message RERRER to the previous node $B$. RERRER informs node $B$ that the route is under local repair and node $B$ is the destination of RERREQ. Node $B$ is required to set timer. If node $B$ cannot receive RERREQ in certain duration, node $B$ should report route error message to source node $A$ and request for source repair. When the route is linked again, the data buffered in node $B$ are forwarded via the fixed route.What mentioned above is in the situation that the link break 
is located at intermediate position of the original path (Fig. 5(a)), the destinations of repair request packets from node $C$ and node $D$ are respectively node $E$ and node $B$. If the break occurs at the end of the link between node $E$ and node $F$ (Fig. 5(b)), the destination of repair request packet is not the node after next but the adjacent node $F$.

\subsection{Simulation Analysis}

We investigate the effect of our route repair method using four performance criterions, such as packet delivery ratio, average end-to-end delay, normalized routing overhead and route discovery count. A comparison is conducted over Competition-based scheme with and without route repair. The data are obtained when the packet rates of communication nodes are respectively 5, 20 and 50 packets/s. The simulation scenario and parameters are the same as the section of simulation model.

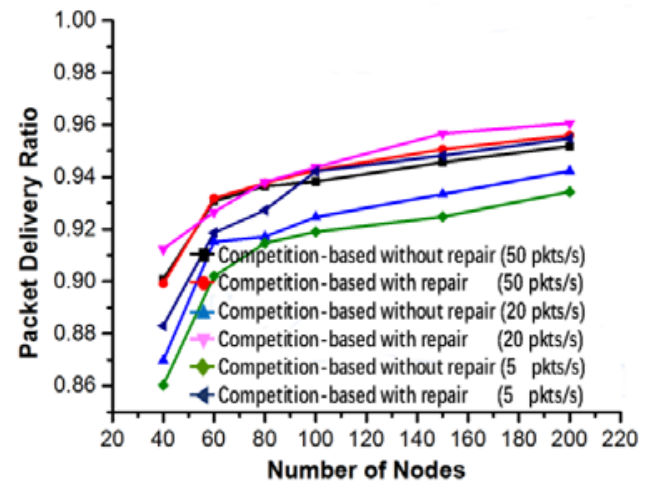

(a)Packet Delivery Radio.

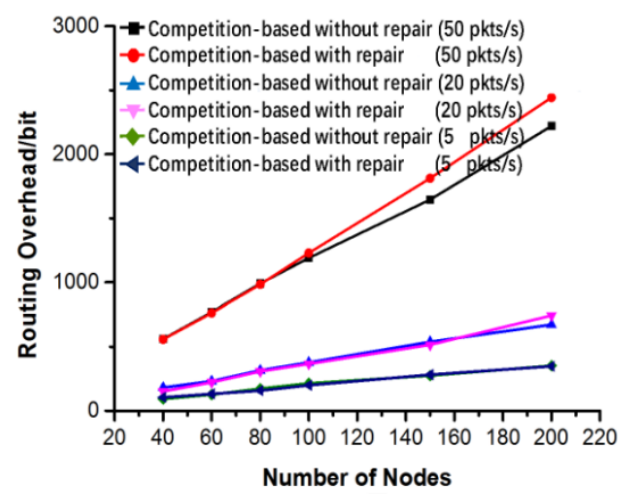

(c)Normalized Routing Overhead..

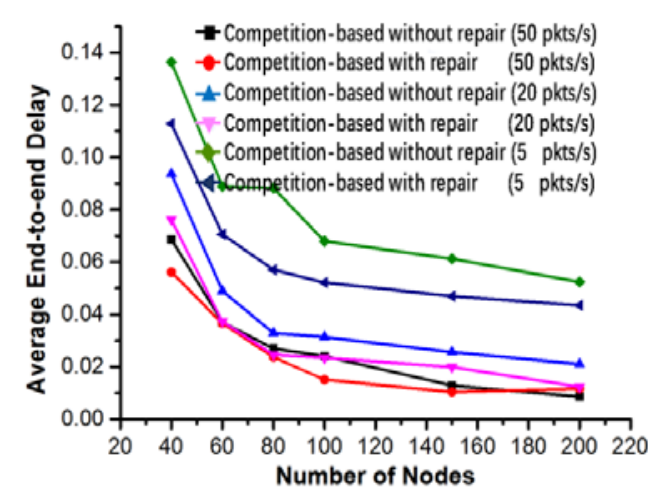

(b)Average End-to-End Delay.

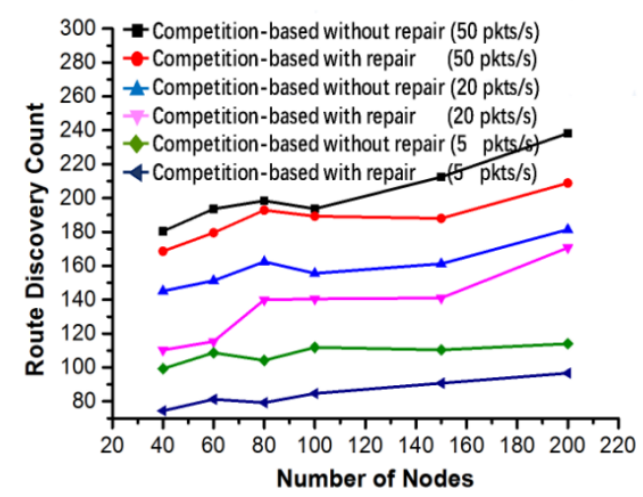

(d)Route Discovery Count.

Fig. 6. Performance of Competition-based with and without route repair.

Fig. 6(a) shows that route repair increases packet delivery ratios across varying number of nodes. Without route repair, once the route is unavailable in certain duration, the intermediate nodes discard data packets. With route repair, the intermediate nodes save 
packets. Once link break is fixed, these packets would be resent and pocket loss is reduced. When network scale expands, available substitute nodes increases. At the time, successful rate of repair also increases and packet delivery ratio finally increase greatly. Fig. 6(b), Fig. 6(c) and Fig. 6(d) shows that route repair gradually decreases average latency of packets delivery, routing overheads and route discovery count across varying number of nodes. With local route repair, it is the intermediate node $\left(N_{u p}\right.$ or $\left.N_{\text {down }}\right)$, not source node, to issue the re-establishment. Due to only 3 hops in local repair, route discovery process is shortened and the repair requests are limited in the surrounding area of breakage. When node density goes up, more intermediate nodes are available to bridge link gaps, hence the latency is largely shortened.

\section{Conclusion}

In the paper, we focus on routing discovery and repair issues for large-scale VANET, and propose a competition-based route algorithm. With the design of forwarding area and forwarding delay, recipients of RREQ compete for becoming the next-hop node, which effectively reduces the production and diffusion of control messages. Using competition scheme, the optimal node with longer link length and residual lifetime is selected, which reduce the negative impact of the expansion of topology and the increase of moving vehicles. And furthermore, one of the upstream and downstream nodes is also selected competitively according to its own stability to establish a only 3 hop short route to fulfill the quick route repair. The simulation results prove that our competition-based scheme is more suitable for VANET in large-scale urban traffic environment.

\section{References}

[1] Li F, Wang Y and Carolina N., "Routing in Vehicular Ad Hoc Networks : A Survey.” IEEE Vehicular Technology Magazine, 12-22, 2 June, 2007. Article (CrossRef Link).

[2] Jung S, Lee S and Oh H., "Improving the Performance of AODV(-PGB) based on Position-based Routing Repair Algorithm in VANET,” KSII Transactions on Internet and Information Systems, 4(6): 1063-1079, 2010. Article (CrossRef Link).

[3] S. Singh and S. Agrawal, "VANET routing protocols: Issues and challenges," in Proc. of Recent Advances in Engineering and Computational Sciences (RAECS) 2014, Chandigarh: 1-5, 2014. Article (CrossRef Link).

[4] Taleb T, Sakhaee E, Member S et al., “A Stable Routing Protocol to Support ITS Services in VANET Networks,” IEEE Transactions on Vehicular Technology, 56, 3337-3347, December 2007. Article (CrossRef Link).

[5] Perkins CE, Park M and Royer EM, “Ad-hoc On-Demand Distance Vector Routing,” in Proc. of IEEE Workshop of Mobile Computer System and Applications, pp. 90-100, 2013.

Article (CrossRef Link). 
[6] Wu Yt, Liao W, Member S et al., "Impact of Node Mobility on Link Duration in Multihop Mobile Networks,” IEEE Transactions on Vehicular Technology, 58(5): 2435-2442, 2009.

Article (CrossRef Link).

[7] Heissenb M, Braun T, Bernoulli T et al., "BLR : Beacon-Less Routing Algorithm for Mobile Ad-Hoc Networks,” Computer Communications, 27(11): 1076-1086, 2004.

Article (CrossRef Link).

[8] Fazio P, Tropea M and Rango FD., “A Novel PER Degradation Model for VANETs,” IEEE Communications Letters, 19(5): 851-854, 2015. Article (CrossRef Link).

[9] Haas ZJ, Member S and Pearlman MR. Zone Routing Protocol. IEEE/ACM Transactions on Networking 2001; 9(4): 427-438. Article (CrossRef Link).

[10] Sun Y, Chen Y and Xu Y. A GPS Enhanced Routing Protocol for Vehicular Ad-hoc Network. In Proceedings of the 2011 IEEE International Conference on Robotics and Biomimetics. ISBN 9781457721380, pp. 2096-2101. Article (CrossRef Link).

[11] Chen, K. H., Dow, C. R., Chen, S. C., Lee, Y. S. and Hwang, S. F., "Harpiagrid: a geography-aware grid-based routing protocol for vehicular ad hoc networks," Journal of Information Science \& Engineering, 26(3): 817-832, 2010.

http://www.iis.sinica.edu.tw/page/jise/2010/201005_06.pdf

[12] Panichpapiboon S and Pattara-Atikom W., "A review of information dissemination protocols for vehicular ad hoc networks,” IEEE Communications Surveys and Tutorials, 14(3): 784-798, 2012. Article (CrossRef Link).

[13] Azzuhri SR, Portmann M and Tan WL., "Evaluation of Parameterised Route Repair in AODV," in Proc. of International Conference on Signal Processing and Communication Systems,2010 4th International Conference on, IEEE, pp. 5709663.1-5709663.7, 2010. Article (CrossRef Link).

[14] Jain J, Gupta R and Bandhopadhyaya TK., "Performance analysis of proposed local link repair schemes for ad hoc on demand distance vector," IET Networks, vol. 3, Issue 2, pp. 129-136, 2014. Article (CrossRef Link).

[15] Qu H., "A New Local Repair Scheme Based on Link Breaks for Mobile Ad Hoc Networks," in Proc. of Seventh Annual Communications Networks and Services Research Conference 2009. ISBN 9780769536491, pp. 364-371, 2009. Article (CrossRef Link).

[16] Minh LV, Mingchuan Y, Liang Y et al., "Congestion-aware and hybrid routing protocol for vehicular ad hoc network," in Proc. of 2nd International Conference on Computer Science and Network Technology, ISBN 9781467329644, pp. 349-353, 29-31 Dec. 2012.

Article (CrossRef Link).

[17] Zhang Q, Zheng H, Lan J et al., "An autonomous information collection and dissemination model for large-scale urban road networks,” IEEE Transactions on Intelligent Transportation Systems, 17(4): 1085-1095, 2016. Article (CrossRef Link).

[18] Hou X, Li Y, Jin D et al., "Modeling the impact of mobility on the connectivity of vehicular networks in large-scale urban environments," IEEE Transactions on Vehicular Technology, 65(4): 2753-2758, 2016. Article (CrossRef Link). 
[19] Liu H, Yang L and Zhang Y., "Improved AODV Routing Protocol Based on Restricted Broadcasting by Communication Zones in Large-Scale VANET,” Arabian Journal for Science and Engineering, 40(3): 857-872, 2015. Article (CrossRef Link).

[20] He J, Cai L, Cheng P et al., "Delay Minimization for Data Dissemination in Large-Scale VANETs with Buses and Taxis,” IEEE Transactions on Mobile Computing, 15(8): 1939-1950, 2016. Article (CrossRef Link).

[21] Cheng J, Cheng J, Zhou M et al., “Routing in Internet of Vehicles: A Review,” IEEE Transaction on Intelligent Transportation Systems, 16(5): 2339 - 2352, 2015. Article (CrossRef Link).

[22] Zhou L, "QoE-Driven Delay Announcement for Cloud Mobile Media," IEEE Transactions on Circuits and Systems for Video Technology, vol. 27, no. 1, pp. 84-94, 2017.

Article (CrossRef Link).

[23] Zhou L, "Mobile Device-to-Device Video Distribution: Theory and Application," ACM Transactions on Multimedia Computing Communications and Applications, vol. 12, no. 3, pp. 1253-1271, 2016. Article (CrossRef Link).

[24] Wang L, Wang Y and Wu C., "A Receiver-Based Routing Algorithm Using Competing Parameter for VANET in Urban Scenarios," Internet of Vehicles - Technologies and Services, pp. 140-149, 2014. Article (CrossRef Link).

[25] Sahu PK, Wu EHK, Sahoo J et al., "BAHG: Back-BoneAssisted Hop Greedy Routing for VANET's City Environments,” IEEE Transactions on Intelligent Transportation Systems, 14(1): 199-213, 2013. Article (CrossRef Link). 


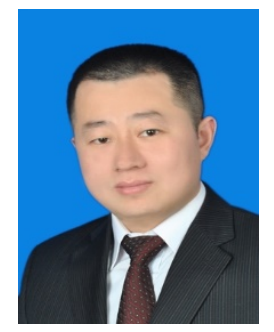

Cheng Wu received the M.Sc. degree in computer engineering from Concordia University in Canada, in 2005, and the Ph.D. degree from Northeastern University, USA, in 2010. Currently, he is an Associate Professor at the School of Urban Rail Transportation, Soochow University, China. His research interests include neural and fuzzy systems, dynamic multi-agent systems, intelligent human-computer interaction and Big Data technology and applications. He authored or co-authored over 20 publications in International Journal of Intelligent and Fuzzy System, International Conference on Autonomous Agents and Multi-agent Systems etc. He was also involved in several projects in which these techniques are being applied in the fields of communication, robotics and computer vision.

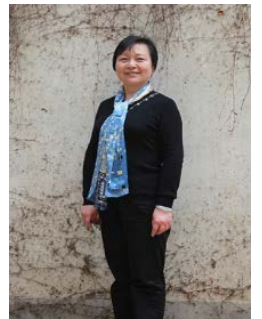

Yiming Wang received the M.Sc. degree in computer engineering from Soochow University, and the Ph.D. degree from Nanjing University of Posts and Communications. Currently, she is a Full Professor at the School of Urban Rail Transportation, Soochow University, China. Her research interests include wireless communications, cognitive wireless sensor network, and intelligent transportation technology and applications. She authored or co-authored over 60 publications in Wireless Personal Communications, Chinese Journal of Electronics and IEEE GlobalSIP Conference etc. She was also involved in several projects in which these techniques are being applied in the fields of communication, robotics and computer vision. She is also an active reviewer for Circuits Systems And Signal Processing and Wireless Personal Communication etc. 